Sociologie et sociétés

\title{
L’alimentation entre plaisir(s) et nécessité(s) en France et aux États-Unis
}

\author{
Quelques variations dans la presse féminine depuis les années
} 1930

\section{Diet Between Pleasure(s) and Necessity(ies) in France and in the United-States}

\section{Some Variations in Women's Magazines since the 1930's}

\section{Faustine Régnier}

Volume 46, numéro 2, automne 2014

Manger - entre plaisirs et nécessités

Eating - Between Pleasure and Necessity

URI : https://id.erudit.org/iderudit/1027143ar

DOI : https://doi.org/10.7202/1027143ar

Aller au sommaire du numéro

\section{Éditeur(s)}

Les Presses de l’Université de Montréal

ISSN

0038-030X (imprimé)

1492-1375 (numérique)

Découvrir la revue

Citer cet article

Régnier, F. (2014). L’alimentation entre plaisir(s) et nécessité(s) en France et aux États-Unis : quelques variations dans la presse féminine depuis les années 1930. Sociologie et sociétés, 46(2), 85-108. https://doi.org/10.7202/1027143ar

\section{Résumé de l'article}

L'article examine la place respective tenue par le plaisir et la nécessité, et leur articulation au fil du temps, à partir d'un corpus de conseils d'économie domestique de 1200 pages, issu de la presse féminine française et américaine (Modes et Travaux et Good Housekeeping) entre 1934 et 2010. Partant d'un examen des termes employés pour indiquer d'une part le plaisir, d'autre part la nécessité, il montre les évolutions, à la fois dans la place tenue par le plaisir et par la nécessité, et dans les domaines où l'un et l'autre s'expriment (passage des contraintes liées au budget et à la satisfaction de besoins alimentaires de base à des injonctions diététiques et individualistes ensuite). S'inscrivant dans les analyses de $\mathrm{N}$. Elias dans $L$ a civilisation des moeurs et $L$ a dynamique de l'Occident, l'article met en évidence que l'expression de la nécessité et la place laissée au plaisir témoignent d'un travail de civilisation, dans lequel la contrainte implicite devient autocontrainte. L'article montre que le plaisir, plus récemment affirmé et mis en valeur, ne s'effectue jamais sans son articulation à la nécessité : un hédonisme, sans doute, mais contraint.
Tous droits réservés ㄷ Les Presses de l’Université de Montréal, 2014
Ce document est protégé par la loi sur le droit d'auteur. L'utilisation des services d'Érudit (y compris la reproduction) est assujettie à sa politique d'utilisation que vous pouvez consulter en ligne.

https://apropos.erudit.org/fr/usagers/politique-dutilisation/ 


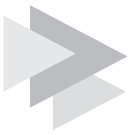

\section{L'alimentation entre plaisir(s) et nécessité(s) en France et aux États-Unis}

Quelques variations dans la presse féminine depuis les années 1930

\author{
FAUSTINE RÉGNIER \\ INRA UR 1303 \\ Alimentation et sciences sociales (Aliss) \\ 65 , boulevard de Brandebourg \\ 94205 Ivry-sur-Seine Cedex \\ France \\ Courriel: Faustine.Regnier@ivry.inra.fr
}

Dassir et nécessité, comment s'articulent ces notions dans un corpus homogène
du point de vue de ses sources (la presse féminine) et de son objet (les normes d'alimentation et de corpulence) ? L'objet de cet article est l'analyse de la place tenue par le plaisir et la nécessité, et des relations établies entre les deux notions. Quand la nécessité est privilégiée, le plaisir peut-il se profiler? À l'inverse, si le plaisir domine, qu'en est-il des nécessités? Trouve-t-on, comme le suggère Épicure (voir introduction de ce numéro), des plaisirs nécessaires? Quelles sont, enfin, les évolutions au fil du temps, à la fois dans la place respective tenue par le plaisir et par la nécessité, et dans les domaines où s'expriment l'un et l'autre ${ }^{1}$ ?

Pour mener cette réflexion, on fera appel aux analyses de N. Elias dans La civilisation des mours et La dynamique de l'Occident. Si les magazines féminins sont un lieu où s'opère une forme de "processus de civilisation» (Giet, 2005), qu'observe-t-on de ce travail de civilisation? Pour N. Elias, le processus de civilisation est fondé à la fois sur

1. L'auteure adresse ses très vifs remerciements au comité de rédaction de Sociologie et sociétés, aux relecteurs anonymes ainsi qu'à N. Vialles et aux membres du séminaire Nourritures, dont les commentaires et suggestions ont permis d'améliorer considérablement la première version de ce texte. Elle est également très reconnaissante à l'équipe du Département «Nutrition, Food Studies and Public Health» de la New York University pour son accueil durant l'année 2011-2012. 
un refoulement des affects et sur le passage de la contrainte à l'autocontrainte: comment ce double mouvement est-il énoncé, dans ce corpus de la presse féminine? Si l'expression des pulsions, des sentiments, des sensations est marquée, au cours du processus de civilisation, par une réserve de plus en plus grande, qu'en est-il du plaisir, qui relève de l'affect? Est-il refoulé, ou bien assiste-t-on au contraire à son expression de plus en plus nette, remarquée ailleurs (Dupuy, 2013)? De même, observe-t-on, au fil du temps, conformément au processus décrit par N. Elias, une transformation des contraintes en autocontraintes, ou a-t-on affaire au contraire à une diminution des contraintes, voire à une forme de «gastro-anomie» (Fischler, 1990)? Enfin, comment peut-on mettre en relation ces évolutions dans la presse et les transformations des sociétés françaises et américaines? L'instauration d'une société d'abondance ( «the affluent society» de Galbraith, 1958) et l'avènement des trente glorieuses (Fourastié, 1979) ont-elles conduit à un effacement des nécessités et au triomphe de l'hédonisme (Mendras, 1988)?

L'expression du plaisir et de la nécessité est analysée dans un vaste corpus de la presse féminine française et américaine. Malgré les critiques dont elle a pu faire l'objet, la presse féminine constitue en effet un objet privilégié où s'expriment modes et tendances, valeurs et normes. Les magazines féminins constituent tout d'abord les canaux privilégiés de diffusion des modes, qu'ils contribuent également à lancer (Besnard et Grange, 1993; Warde, 1997). Ensuite, les magazines féminins sont à la fois des vecteurs de diffusion des normes, garants des valeurs dominantes (Bonvoisin et Maignien, 1986), mais ils sont également le reflet - et parfois peut-être le moteur? - de l'émancipation des femmes, en particulier à l'égard des contraintes domestiques. Les revues féminines peuvent ainsi être considérées comme une caisse de résonance des évolutions de la société.

Ce travail s'inscrit dans une recherche, plus large, s'intéressant aux normes d'alimentation et de corpulence en France et aux États-Unis, dans une perspective rétrospective. Il est fondé sur l'analyse détaillée entre 1934 et 2010 de deux revues féminines publiées de part et d'autre de l'Atlantique, Modes et Travaux et Good Housekeeping 2 . Ces deux revues ont été retenues en raison de leur ancienneté - laquelle permet une étude dans la longue durée - et de leurs forts tirages, parmi les plus importants de la presse féminine. Modes et Travaux, créée en 1919, et Good Housekeeping créée en 1885, sont parmi les revues les plus lues en France et aux États-Unis avec un tirage mensuel moyen, en 2011, de 599272 exemplaires pour Modes et Travaux et de 4336711 exemplaires pour Good Housekeeping. Il s'agit donc d'un phénomène de masse, et l'on a montré, à propos de l'intégration de cuisines étrangères, comment les revues féminines

2. L'année 1934 constitue en effet la date à partir de laquelle les numéros de Modes et Travaux sont disponibles à la BNF. La même date de départ a donc été retenue pour Good Housekeeping dont tous les numéros ont été dépouillés à la New York Public Library. Pour ce travail, ont été pris en compte, par une lecture systématique de tous les numéros, toutes les rubriques, textes et articles qui permettaient de cerner des modèles alimentaires, définissant à la fois une «bonne alimentation» et donnant les mesures du « corps idéal». 
permettent de cerner des modèles nationaux en matière d'alimentation (Régnier, 2004).

Dans un contexte d'intense «médiatisation du culinaire» (Boutaud et Madelon, 2010), ces magazines ont également été retenus pour la place spécifique qu’ils accordent aux conseils d'économie domestique: ce sont deux revues généralistes qui présentent l'avantage de n'être pas exclusivement centrées sur la mode. Toutes deux diffusent savoirs et normes en matière d'éducation domestique — aux États-Unis, les «Home Economics » - et elles abordent, au fil des numéros, tous les thèmes relatifs à l'alimentation, la nutrition, la cuisine, l'hygiène, le budget, les soins aux enfants et la santé. Enfin, ces revues s'adressent toutes deux à la classe moyenne, c'est-à-dire à la cible privilégiée, aux États-Unis, des campagnes d'éducation (Levenstein, 1988; Bruegel, 2013) et, pour la France, à une fraction de la population particulièrement sensible à toutes les recommandations liées à l'alimentation (Régnier et Masullo, 2009).

Tous les numéros de ces deux revues depuis janvier 1934 ont été systématiquement consultés jusqu'en décembre 2010. Un corpus de 1200 pages de notes a été constitué (124311 occurrences pour Modes et Travaux, 291971 occurrences pour Good Housekeeping). Il a fait l'objet d'une analyse de contenu classique et d'une analyse textuelle menée à l'aide du logiciel Hyperbase, à partir d'une grille systématique d'analyse. L'originalité de notre travail se situe dans la collecte systématique d'un vaste matériel discursif ${ }^{3}$. Les illustrations (dessins puis photographies) de même que les publicités n'ont pas été prises en compte: leur analyse relève d'une méthodologie autre que celle de l'analyse textuelle. Cependant, nous y avons prêté attention chaque fois qu'il a été possible, en particulier dans les premières années du corpus où faute d'avoir des discours détaillés, il nous a fallu prendre en compte les publicités, qui tiennent une place très importante à cette date.

On étudiera ainsi dans un premier temps la place respective des termes "plaisir» et «nécessité» dans le corpus, ainsi que celle de leurs synonymes. Nous analyserons ensuite les domaines d'expression de la nécessité avant de montrer comment cette nécessité, d'explicite au départ, devient au fil du temps une nécessité implicite. Dès lors, elle fait plus de place au plaisir, mis en avant dans les années récentes: la contrainte aurait-elle disparu?

\section{LES EXPRESSIONS DU PLAISIR ET DE LA NÉCESSITÉ}

\section{a) «Plaisir(s) » et « nécessité(s)»}

L’analyse des occurrences des termes «plaisir(s)» et «nécessité(s)» fait apparaître à la fois des éléments communs à la France et aux États-Unis, et spécifiques à chacun des corpus.

3. Il aurait été intéressant de croiser d'autres types d'analyse, comme celles relevant de la sémiopragmatique - qui auraient permis de mieux prendre en compte les dispositifs de communication — ou de mettre l'accent sur la dimension proprement féminine de ce secteur de la presse (Damian-Gaillard et al., 2009). Ces analyses restent à mener. 
Tableau 1. Plaisir(s) et nécessité dans Modes et Travaux et Good Housekeeping

\begin{tabular}{|l|l|l|}
\hline & Modes et Travaux & Good Housekeeping \\
\hline Plaisir (pleasure) & 82 & 21 \\
\hline Plaisirs (pleasures) & 10 & 6 \\
\hline Nécessité (necessity) & 11 & 7 \\
\hline Nécessaire(s) (necessary) & 56 & 111 \\
\hline
\end{tabular}

Dans les deux revues, le substantif même «plaisir(s)» est plus fréquent que le substantif «nécessité» (tableau 1). La «nécessité» est peu présente dans les conseils d'économie domestique, dont elle ne fait pas réellement partie par son côté abstrait. Le goût de «nécessité» cher à Bourdieu relève du vocabulaire sociologique, mais non de l'éducation ménagère telle qu'elle se présente dans les revues. En revanche, l'adjectif «nécessaire», plus fréquent dans le langage commun, est beaucoup plus utilisé.

De là une première différence importante entre la France et les États-Unis: le discours lié à la nécessité est plus important outre-Atlantique, alors que le plaisir est une notion française, tout particulièrement si l'on rapporte ses occurrences à l'ensemble des corpus. C. Fischler et E. Masson, dans leur comparaison internationale des attitudes relativement à l'alimentation (Fischler et Masson, 2008), ont bien souligné l'importance du plaisir dans les discours des interviewés francophones. Aux États-Unis, le plaisir se fait singulièrement rare!

Ce plaisir est plus souvent exprimé au singulier qu'au pluriel, notamment en France. La préférence pour le singulier semble indiquer une forme d'essentialisation du plaisir : plutôt que d'évoquer le plaisir dans ses différentes manifestations, Modes et Travaux s'intéresse au plaisir en soi. L'usage du pluriel pourrait suggérer un degré moindre dans le plaisir, le pluriel venant en quelque sorte disperser ou diluer l'intensité du plaisir.

D'un point de vue chronologique (annexe 1), les apparitions du «plaisir» dans le corpus français sont rares jusqu'aux années 1980 (13 occurrences entre 1934 et la fin des années 1970). C'est à partir des années 1980 qu'il devient plus fréquent, passant subitement à 10 occurrences dans cette décennie, puis à 13 dans les années 1990 et 46 dans les années 2000, dont il est une spécificité lexicale $(5,6)^{4}$. De la même façon, les «plaisirs», rares, apparaissent tardivement (première occurrence en novembre 1978), et ils sont majoritaires dans les discours des années 2000 (5 occurrences sur 10). Au total, sur les 82 occurrences de plaisir(s), 51 apparaissent dans les années 2000.

4. Le logiciel Hyperbase, créé par Étienne Brunet, permet d'établir des tables de spécificité lexicale pour chaque décennie, dans chacune des revues. Les listes de spécificités présentent les formes excédentaires, celles dont l'écart réduit est supérieur à 2. Hyperbase compare les fréquences observées aux fréquences telles qu'elles seraient théoriquement observées si les mots étaient répartis aléatoirement dans l'ensemble des textes du corpus (pour le calcul de ces écarts réduits, voir Régnier, 2004). Quand un terme est propre à une décennie particulière, nous indiquons entre parenthèses la valeur de son écart réduit. 
Dans le corpus américain, on n’observe pas cet intérêt accru pour le plaisir dans la décennie actuelle. Le terme "pleasure», rare, est relativement plus fréquent dans les années 1950 que dans les discours contemporains (annexe 2).

La «nécessité» est tellement rare que son emploi est aléatoire. En France comme aux États-Unis, seules les années 1950 font apparaître un emploi plus fréquent (5 occurrences sur les 11 au total dans Modes et Travaux; 4 sur 7 au total dans Good Housekeeping). En ce qui concerne l'adjectif «nécessaire», plus fréquent, le seul élément remarquable dans Modes et Travaux est sa surprésence statistique, aussi bien au singulier qu'au pluriel, dans les années 1950: dans cette décennie, le discours de la nécessité est plus marqué. Aux États-Unis, les évolutions sont plus claires: l'usage de l'adjectif «necessary» connaît une remarquable diminution dans le corpus tout au long de la période étudiée. En surfréquence dans les années 1930 - de façon statistiquement significative - , il connaît ensuite une diminution régulière de son emploi, de plus en plus marquée jusqu'à nos jours, et son déficit est significatif dans les années 1990 et 2000. S'agit-il d'une diminution de la contrainte dans la période contemporaine? Ou bien faut-il chercher d'autres formulations de la nécessité?

\section{b) Les autres expressions du plaisir et de la nécessité}

Le plaisir et la nécessité peuvent être exprimés par d'autres termes, révélés par l'analyse du corpus ${ }^{5}$.

Dans le champ du plaisir, différents termes apparaissent, qu'il s'agisse du même champ lexical, ou qu'il s'agisse de termes synonymes qui émergent par leur fréquence d'emploi:

\section{Tableau 2. Autres formulations du plaisir dans Modes et Travaux et Good Housekeeping}

\begin{tabular}{|l|l|l|}
\hline & Modes et Travaux & Good Housekeeping \\
\hline Plaire / to please & 7 & 10 \\
\hline Plaisant / pleasant & 2 & 12 \\
\hline Aimer / to love & $42^{6}$ & 105 \\
\hline Enjoy & & 111 \\
\hline
\end{tabular}

Le verbe «plaire» et l'adjectif «plaisant» sont rares, comparés aux occurrences du «plaisir». Le verbe «aimer» est plus fréquent: c'est une façon, certes indirecte, de capter le plaisir, mais il désigne bien l'appétence pour tel ou tel aliment, ou le plaisir pris à tel ou tel repas. D'un point de vue chronologique en France comme aux États-Unis

5. Analyse des spécificités propres à chaque décennie (années 1930; années 1940; etc. jusqu'aux années 2000) et analyse de l'évolution lexicale (termes dont l'emploi diminue ou augmente au fil du temps).

6. Il s'agit du lemme du verbe «aimer» c'est-à-dire le verbe dans toutes ses déclinaisons. 
(annexes 1 et 2), le verbe connaît une très forte expansion dans les années 2000, où il est en excédent statistique dans les deux corpus. Dans Good Housekeeping en particulier, le verbe « to love» montre une expansion remarquable et continue au fil du temps: c'est le terme qui connaît la plus forte progression au fil du temps. En déficit statistique dans les années 1930 et 1940, son emploi est de plus en plus fréquent jusqu'à connaître l'excédent statistique dans les années 1990 et 2000: peut-on y lire l'expression d'une forme de plaisir dans le corpus américain, qui rejoindrait la place prise par le "plaisir» en France dans les années contemporaines?

«To enjoy», enfin, est une manière caractéristique du corpus américain d'indiquer le plaisir. Ses occurrences sont croissantes jusqu'aux années 1970, dont il est spécifique $(5,3)$, puis le relais est pris par le verbe «to love».

En ce qui concerne la nécessité, plusieurs termes sont également signifiants: les verbes d'injonction ( «devoir», «falloir»), mais aussi tous les termes qui indiquent ce qui doit être, et qui soulignent le caractère nécessaire de tel ou tel aliment, tel repas ou tel régime («principe(s)» et «règle(s)», ainsi que l'adjectif « essentiel»).

\section{Tableau 3. Autres formulations de la nécessité dans Modes et Travaux et Good Housekeeping}

\begin{tabular}{|l|l|l|}
\hline & Modes et Travaux & Good Housekeeping \\
\hline Devoir (must) & 238 & 225 \\
\hline Falloir (have to, has to) & 234 & 123 \\
\hline Devrait ${ }^{8}$ (should, ought to) & 17 & 530 \\
\hline Règle(s) (rules) & 70 & 77 \\
\hline Principes (principles) & 47 & 25 \\
\hline Essentiels (essentials) & 54 & 174 \\
\hline
\end{tabular}

La nécessité exprimée par le verbe «devoir» et ses déclinaisons est particulièrement apparente. Ces verbes d'injonction sont ainsi des vecteurs de la transmission de règles et de normes. Par exemple, parlant de la mère de famille, Good Housekeeping écrit: «Elle devrait connaître quels sont les aliments qui sont essentiels à une bonne santé» $(09 / 49$, p. 28).

En France, cette injonction prend la forme d'un impératif: les verbes «devoir» et «falloir» sont employés essentiellement au présent, donc dans un sens fort, et beaucoup moins au conditionnel. Aux États-Unis, «must» - qui marque une injonction forte - est moins employé que «should» — signalant une injonction moins marquée, plutôt sur le mode du conseil: respectivement 225 et 519 occurrences ${ }^{9}$.

7. Toutes les formes de «devoir» au présent pour le corpus français.

8. Toutes les formes de «devoir» au conditionnel pour le corpus français.

9. Et dans une très moindre mesure « ought to» (11 occurrences). 
D’un point de vue chronologique (annexes 1 et 2), dans Modes et Travaux, les termes indiquant une injonction forte ( «doit», «devons», «règle», «principe») connaissent un pic de fréquence dans les années 1950: «doit» $(3,4) ;$ «devons» $(3,0)$, «nécessité » $(2,9)$; « essentielle» $(2,8)^{10}$; «nécessaire» $(2,7)$ et «normes» $(2,6)$ sont ainsi des spécificités de cette décennie, où il se confirme que le discours de la nécessité est particulièrement marqué. Après cette date, ces termes sont en forte régression.

Voilà un point commun avec Good Housekeeping: la diminution chronologique du discours injonctif et son déficit dans les années contemporaines. Ainsi, les verbes d'injonction «must» et «should» sont propres aux années 1940 et 1950 (ainsi qu'aux années 1930 pour «must»), puis ils connaissent un déclin jusqu'à aujourd'hui et atteignent le déficit statistique. L'adjectif «essential» connaît quant à lui une forte diminution à partir des années 1970, avec un déficit de nos jours.

Pour autant, dans la décennie 2000, le discours de la nécessité ne disparaît pas totalement: dans Modes et Travaux, le verbe «falloir» continue d'être utilisé, et dans Good Housekeeping, des «règles» sont régulièrement énoncées durant cette décennie. Mais ces termes ne sont pas propres aux discours de la période.

Quels sont alors les grands domaines où s'exprime la nécessité? Quels sont les éléments qui présentent un caractère de nécessité et d'obligation?

\section{NÉCESSITÉS ET PLAISIRS NON NÉCESSAIRES}

Si manger est bien un plaisir nécessaire, quelles sont, à travers ces différents termes, les grandes «nécessités» exprimées par les revues? Quels sont les différences ou points communs entre la France et les États-Unis, et leur évolution au fil du temps?

\section{a) La «nécessité» au sens propre: les questions de budget}

La «misère» est bien le sens premier de nécessité. Dans ces revues, qui ne s’adressent pas à des «nécessiteux», et qui ont pour but de divertir, il n'est évidemment pas question de pauvreté. Mais il y est bien question de budget à surveiller et à équilibrer: l'un des buts premiers des conseils d'économie domestique.

Cette thématique est présente tout au long du corpus dans les deux magazines. La question du coût fait partie des conseils d'économie domestique donnés par les revues: elles s'inscrivent, côté américain, à la suite des travaux d'Atwater et des nutritionnistes au début du $\mathrm{xx}^{\mathrm{e}}$ siècle (Levenstein, 1988), et côté français, dans une optique d'alimentation rationnelle, diffusée en France sous l'égide de la Société scientifique d'hygiène alimentaire et d'alimentation rationnelle de l'homme ${ }^{11}$, et qui vise à corriger «à la lumière des acquis récents de la nutrition humaine, les habitudes alimentaires et économiques des ouvriers » (Bruegel, 2013: 30). Il s'agit d'apprendre à la mère de famille à prendre en compte tout à la fois la valeur nutritionnelle et le coût des aliments. Les

10. La présence de l'adjectif «essentiels» dans les années 2000 ne renvoie pas à un discours de la nécessité: il désigne les «acides gras essentiels», en vogue ces années-là.

11. Sur ces questions, on se reportera avec profit aux travaux de T. Depecker (2014). 
revues s'attachent ainsi à mettre en valeur des plats ou des aliments économiques qui assurent à la fois la gestion efficace du budget et la bonne santé de la famille.

Des solutions de rechange bon marché - en particulier en remplacement de la viande - sont ainsi proposées: «Souvenons-nous toujours que le prix et la valeur marchande d'un aliment ne sont pas proportionnels à leur valeur nutritionnelle» (MT, 04/1982, p. $226^{12}$ ).

En outre, il s'agit pour les revues de guider les femmes dans le monde nouveau de la consommation de masse. C'est en effet au tournant $\mathrm{du} \mathrm{xx}^{\mathrm{e}}$ siècle que la discipline des «Home economics» apparaît outre-Atlantique, au moment où l'Amérique entre dans l'ère de l'abondance. Les «Home economics» visent alors à apprendre aux femmes comment être à la fois d'efficaces ménagères et de bonnes consommatrices, à leur montrer comment opérer des choix judicieux (Goldstein, 2012). Dans une optique d'empowerment, les revues fournissent guides et repères de consommation.

Mais cette question du budget alimentaire, du coût des aliments, ce souci d'économie au quotidien font l'objet d'une attention plus particulière à certaines époques, différentes en France et aux États-Unis. En France, on peut distinguer deux moments. Dans les années 1950 et 1960 tout d'abord, où la question de la nécessité budgétaire s'impose plus largement à travers les adjectifs «économique(s)» $(2,3$ et 2,8$)$ et «onéreux» (2,6 pour les années 1960). À cette époque en effet, la revue fait une très grande place à la diététique, et plus particulièrement à la présentation des principes d'une alimentation rationnelle qui concilie souci budgétaire et nutritionnel.

Dans les années 1970, cette thématique financière passe au second plan, mais elle réapparaît dans les années 1980, à travers l'importance prise par le terme «prix» $(4,1)$, reflet sans doute de la période d'inflation que traverse la France à cette époque, après le choc pétrolier de 1973 qui signe la fin des trente glorieuses (Fourastié, 1979), sans atteindre toutefois l'intensité de la crise économique américaine.

Outre-Atlantique, en effet, la crise est particulièrement brutale, et Good Housekeeping se fait l'écho d'un véritable choc économique. La nécessité budgétaire est prédominante dans les années 1970, où tenir son budget devient une priorité. Bien des termes liés aux questions budgétaires sont propres à cette décennie: «cost» $(8,5)$, «dollar» $(5,2)$; «expensive» $(4,9)$; «money» $(4,4)$; «budget» $(4,2)$; «inexpensive» $(5,2)$; «economical» (4). La revue annonce par exemple en couverture, en avril 1976, un dossier «Ce que l'Amérique aime manger: des plats basses calories, à faible coût» (GH, 04/1976, p. 196). La nécessité est triple: proposer des plats pauvres en calories, à moindre coût, mais qui plaisent également du point de vue du goût. De la même façon, en octobre 1977, la revue proposera de «délicieux repas basses calories pour moins de un dollar par portion» (GH, 10/1977, couverture) : s'il est prédominant, le souci d'économie n'efface pas les préoccupations nutritionnelles.

12. Quand nous citons un extrait de revue, nous indiquons entre parenthèses la revue (MT pour Modes et Travaux, GH pour Good Housekeeping), la date de parution du numéro et la page de la citation. 
Ce souci d'économie domestique est moins apparent dans les années 1990 et 2000, laissant la place à l'expression d'autres contraintes.

\section{b) Les contraintes de temps}

Quand il s'agit d'alimentation, le temps constitue une contrainte forte pour les femmes, en particulier depuis leur arrivée sur le marché du travail. Ce souci du temps est plus prononcé aux États-Unis qu'il ne l'est en France, quand on considère les différentes occurrences des termes qui s'y rapportent. Le terme «temps » apparaît à 438 reprises aux États-Unis, contre 105 en France. D’autres termes indiquent un souci de temps: en France, «rapide(s)» (46 occurrences), «rapidement» (36), «vite» (77), «pressée $(s) »(7)$. Aux États-Unis « fast $^{13}$ » $(173)$ « quick» (132) « quickly» (50), «hurry» (51). Au total, ce sont 844 mentions du temps et de la rapidité aux États-Unis contre 271 en France, soit bien plus chez nos voisins américains, y compris rapporté à la longueur des textes recueillis.

Ce souci de la rapidité aux États-Unis est souligné par d'autres études qui montrent la diminution du temps passé aux États-Unis à faire la cuisine et à consommer, et mettent en évidence le souci de rapidité qui caractérise les évolutions dans le domaine alimentaire aux États-Unis, comparé à la stabilité française pour le temps consacré aux affaires culinaires (Warde et al., 2007). Pour autant, ce souci n'est pas absent du corpus français, tout en étant moins présent.

Ce souci du temps est croissant dans les deux revues depuis les années 1930 mais selon un rythme très différent. En France, sa présence est stable jusqu'aux années 1960, en baisse dans les années 1970 qui sont centrées sur la diététique, puis en augmentation nette. Aux États-Unis, la pente est beaucoup plus forte. Certes, dans les années 1970 et 1980 la revue s'intéresse essentiellement aux contraintes budgétaires, mais le thème de la rapidité reprend de l'importance dans les années 1990 et devient majeur dans les années 2000. Cependant, ce souci se rapporte moins aux questions culinaires proprement dites qu'aux différents régimes amaigrissants dont il s'agit d'obtenir des résultats rapidement.

Pour autant, la rapidité possède dans les deux corpus un statut ambivalent. On trouve tout d'abord une valence positive. Celle-ci concerne les préparations culinaires vantées par les revues - qui cherchent à aider les femmes dans leurs tâches domestiques - en raison de leur rapidité. Entre les années 2006 et 2010, Good Housekeeping édite une rubrique «Healthy in a hurry» qui propose des recettes saines, mais qui peuvent être préparées rapidement. Pour la revue, il s'agit bien de concilier une contrainte spécifique — le temps — avec un objectif de santé et un souci d'économie. Il en va de même pour Modes et Travaux qui régulièrement propose des repas « rapides et faciles à préparer ».

La valence positive de la rapidité s'exprime également par la question d'un amaigrissement rapide: «Aussitôt pris aussitôt perdus... les petits kilos superflus dus aux

13. En ne comptant évidemment pas le terme «fast» quand il désigne le jeûne. 
repas de fin d'année disparaitront rapidement si vous réagissez tout de suite », explique Modes et Travaux (MT, 02/1996, p. 36); «Ce qui est important, c'est de perdre les kilos superflus — vite! » répond en écho Good Housekeeping (GH, 11/1983, p. 240).

Mais dans le même temps, la rapidité présente une valence négative: les deux revues critiquent tel ou tel régime justement parce qu'il est trop rapide. «Souvenezvous, les kilos perdus trop vite se rattrapent la plupart du temps très rapidement» (MT, 10/1983, p.214) explique Modes et Travaux. «Si vous essayez de perdre trop vite trop de poids, vous risquez de programmer une rapide reprise de poids juste après», met en garde Good Housekeeping (GH, 07/2010, p.43).

Aux États-Unis, la rapidité concerne enfin la restauration rapide. Les fast-foods sont régulièrement évoqués par Good Housekeeping à partir des années 1970. Partant du constat que les fast-foods sont partie intégrante du mode de vie américain, la revue teste les différentes chaînes, évalue leur qualité nutritionnelle et fournit ensuite des conseils aux lectrices pour intégrer leur fréquentation dans une alimentation équilibrée. De même, elle propose très régulièrement une version modifiée des plats typiques servis dans les fast-foods, mais adaptés aux exigences nutritionnelles: «Le régime fastfood: gardez les frites, perdez les kilos» (GH, 07/2003, couverture).

\section{c) Diététique et nutrition}

Le respect des règles diététiques ou nutritionnelles constitue un réel impératif dans les deux corpus. Ce souci nutritionnel est à la fois plus marqué et plus précoce aux ÉtatsUnis, où le souci de santé à travers l'alimentation est plus important qu'ailleurs (Fischler et Masson, 2008) : parler d'alimentation aux États-Unis, c'est parler de nutrition. Good Housekeeping se fait fidèlement l'écho des différentes politiques en matière de nutrition. Par exemple, elle rend compte des recommandations nutritionnelles formulées lors de la Conférence nationale de nutrition pour la défense de 1941 lors de laquelle sont présentées les «Recommended Daily Allowances». Elle explique à ses lectrices que veiller à la bonne santé de la famille est un devoir patriotique - dans un contexte plus large où la nutrition relève de la défense nationale (Levenstein, 1993; Bentley, 2002) —, à travers la métaphore du front domestique: «Vous devez, comme un général, planifier votre stratégie en pleine connaissance des besoins de votre ligne de défense» (03/42, p. 104).

Elle relaie ensuite les différentes recommandations du US Department of Agriculture (USDA) : en juillet 1943, le guide nutritionnel fondé sur sept aliments de base — «the Basic Seven ${ }^{14}$ » à consommer tous les jours, qui sont réduits à quatre en $1956^{15}$ et présentés par Good Housekeeping à de très nombreuses reprises ${ }^{16}$. Puis la revue expliquera la pyramide alimentaire mise au point en 1992 par la USDA.

14. Légumes verts et jaunes; agrumes, tomates et légumes crus; pommes de terre et fruits; lait et produit laitiers; viande, poisson, œufs et légumes secs; pains et céréales; beurre et margarine.

15. Fruits et légumes; laitages; viandes et autres protéines; produits céréaliers.

16. Le terme «basic» est un marqueur des années $1960(4,6)$ et $1970(6,8)$. 
Dans ce cadre, le terme «excellent», loin d'indiquer l'excellence gustative comme on pourrait s'y attendre, ou du moins comme un Français pourrait s'y attendre, se réfère à l'excellence des qualités nutritionnelles de tel ou tel aliment, excellente source de calcium (ou de vitamine, ou d'énergie).

$\mathrm{Au}$ fil du temps, la présentation des différents éléments nécessaires à l'équilibre nutritionnel s'efface derrière un souci de santé plus général: dans le corpus américain, tous les termes se rapportant à la santé prennent de plus en plus d'importance et marquent une progression ${ }^{17}$.

En France, ce discours nutritionnel ne devient une nécessité - présentée comme telle - qu'à partir du milieu des années 1950, au moment où la revue crée une rubrique "Savoir s'alimenter», où apparaissent les premiers conseils diététiques (octobre 1952). Cette rubrique change ensuite régulièrement de nom, mais c'est elle qui donnera le plus d'importance à la nécessité de suivre les conseils en matière de diététique: «Seule une alimentation rationnelle vous fournira la clef de la bonne santé », affirme la revue en juillet 1965 .

À ce sujet, conformément à son orientation et à son style, Modes et Travaux procède à une démarche didactique, présentant les grands "groupes d'aliments», leur «valeur», ainsi que l'adaptation de l'alimentation quotidienne aux différentes populations distinguées en fonction de leur âge, de leur activité ou de leur corpulence: à chacune correspond une «bonne ration». Le suivi de ces «règles» ou de ces «principes» diététiques, qui viennent définir une alimentation idéale, est présenté comme une véritable nécessité: les fruits et légumes en hiver «ne sont pas un luxe, mais une nécessité» (MT, 01/1958, p.72).

Dans les années 2000 en France, la diététique — qui s'efface dans les années 1980 et 1990 derrière le souci de minceur, on le verra — retrouve un regain d'intérêt à travers la «nutrition», signe peut-être d'une médicalisation accrue de l'alimentation. La nutrition devient un marqueur de la décennie 2000 («nutrition» 11,4, «nutritionniste» 5,8) et elle est à l'origine d'un discours qui se veut de plus en plus scientifique: «oméga » $(9,7)$, «antioxydants» $(5,2)$, mais aussi «polyphénols» $(3,1)$, «probiotiques» $(2,8)$ font leur apparition et marquent les discours.

\section{d) Maigrir: une absolue nécessité}

C'est un aspect particulier de la diététique: maintenir un poids qui soit dans les normes, ce qui se traduit le plus souvent en souci de minceur qui, dans les années récentes, est devenu la nécessité principale. Il ne s'agit désormais plus d'assurer une alimentation suffisante en quantité, il convient au contraire de veiller à l'excès et de contrôler le poids. Ce passage de la «peur de manquer aux dangers de l'obésité» a en particulier été analysé à propos des aliments pour enfants: avec le temps, le risque n'est plus celui d'une alimentation insuffisante, mais d'une alimentation trop riche (De Iulio, 2011).

17. «Health», «healthfully», «healthy», «healthier». 
Ce souci de minceur est présent, cependant, dès les premières années. La conformité aux canons esthétiques — même variables au fil du temps — est évidemment une nécessité relayée par les magazines féminins. Maigrir, plus précisément, est une préoccupation ancienne, associée à la fois à la recherche de la beauté, mais aussi de la santé.

C'est à partir des années 1950 aux États-Unis et 1960 en France que le thème prend de l'importance. Pour les États-Unis, P. Stearns a montré que le souci de minceur, dont témoignent en particulier les membres de la classe moyenne, s'intensifie certes durant l'après Seconde Guerre mondiale mais trouverait ses origines au tournant du xx ${ }^{\mathrm{e}}$ siècle (Stearns, 1997). Dans Good Housekeeping, c'est véritablement à partir des années 1950 que perdre du poids prend de l'importance, tandis que passent au second plan les autres préoccupations liées à l'économie domestique. Dans ces années 1950, «reducing» $(8,7)$, «calorie» $(4,2)$, «(weight) watchers» $(6,7)$, et «low» dans les décennies suivantes, sont spécifiques, comme le sont les mensurations des différentes parties du corps qui doivent être en conformité avec les normes énoncées: «Quelles doivent être vos mensurations?» (GH, 03/1949, p. 74) demande la revue avant de fournir la liste des critères à remplir. Par la suite, tous les termes se rapportant à la minceur indiquant la mesure du poids ou la perte de poids sont en progression dans la revue, constituant un thème de plus en plus présent ${ }^{18}$. Enfin, les termes indiquant la nécessité comme les «règles» ou les «principes» se rapportent désormais au régime amaigrissant.

En France, ce mouvement s'observe à partir du milieu des années 1960: à compter d'avril 1967, la rubrique «Diététique» de la revue, qui traite de sujets variés, s'assortit systématiquement de «menus basses calories», puis de «recettes basses calories». Au fil du temps, la question de la minceur prend de plus en plus de place. Elle devient dominante dans la revue après mai 1988, date du premier numéro spécial de Modes et Travaux consacré dans son entier à la minceur.

Cette focalisation sur le poids à la fin des années 1980 peut paraître tardive. S'agit-il d'un retard de la France sur les États-Unis et d'une influence américaine comme dans d'autres domaines, musique ou mode vestimentaire par exemple, témoignant d'une forme d'américanisation (Dard et Lüsebrink, 2008)? En ce qui concerne la corpulence, l'hypothèse semble difficile à confirmer. P. Stearns (op. cit.), par exemple, indique que le souci de contrôle du poids aurait émergé plus précocement en Europe, notamment en France, qu'aux États-Unis. S’il s'agit en revanche plus spécifiquement de l'engouement pour tel ou tel régime amaigrissant venu des États-Unis (régime Hollywood, régime Scardale, etc.), dont les revues se font l'écho, il s'agit sans doute d'un alignement d'une revue française sur sa consœur américaine.

Plus largement, ce retard de Modes et Travaux est conforme à ce que l'on observe pour les autres mouvements de mode, qui y apparaissent avec dix à vingt ans de retard par rapport à des revues plus modernes - comme Marie Claire ou Elle - sur lesquelles Modes et Travaux finit par s'aligner (Régnier, 2004). Cette omniprésence du

18. Ce thème n'est pas l'objet du présent article mais est l'objet d'analyses en cours. 
thème de la perte de poids, des régimes et des kilos en trop s'inscrit également dans le souci de modernisation qui anime la revue à partir des années 1990.

Et, comme dans Good Housekeeping, tous les termes indiquant la nécessité se réfèrent désormais exclusivement à la minceur et à la perte de poids: «Une semaine de menus pour perdre sans efforts un ou deux kilos! Et aussi des astuces minceur et nos treize principes de base à respecter absolument» (MT, 12/99, p. 160).

La nécessité porte ainsi sur des domaines divers évoluant au fil du temps. Au début du corpus, cette nécessité est centrée sur les questions de budget: dans le monde de l'après-guerre, il s'agit de gérer des ressources encore rares, avant le passage à une société d'abondance. Dans les années 1950, la France se situe encore dans une période de pénurie ${ }^{19}$ : l'objectif est d'assurer les besoins alimentaires de base et de veiller à ce que l'alimentation fournisse les éléments nécessaires au bon fonctionnement du corps. Dès lors, cette nécessité peut correspondre au premier type de plaisirs distingués par Épicure: garantir la satisfaction d'un plaisir naturel et nécessaire, celle de l'alimentation de base.

La prospérité économique des trente glorieuses, qui s'accompagne en France et aux États-Unis d'une élévation du niveau de vie, du développement des dépenses de loisirs (Galbraith, 1958; Fourastié, 1979) — dont participe l'intérêt pour les pratiques culinaires et la gastronomie (Ferguson, 2004) —, voit alors se développer de nouvelles nécessités: la nutrition, la santé, la minceur. Conformément à la loi d'Engel, la hausse du niveau de vie engendre une baisse de la part consacrée à l'alimentation de base, dans un contexte où les bouleversements sociaux et politiques des années 1960 aux ÉtatsUnis et en France conduisent à remettre en cause les idéaux de la société d'abondance et de la consommation de masse. La préoccupation pour la qualité plutôt que pour la quantité prend alors son essor. Les plaisirs naturels liés à l'alimentation ne relèvent plus du nécessaire, mais les revues, en soulignant leur caractère impérieux, tentent de les élever au rang de plaisirs nécessaires, illustrant la tension propre au domaine culinaire entre besoin et plaisir, nécessité et désir (Boutaud, 2005).

\section{AUTOCONTRAintes ET MONTÉE EN PUISSANCE DU PLAISIR}

L'évolution du vocabulaire vient suggérer, dans les discours ici recueillis, un passage de la contrainte extérieure - explicitement formulée — à l'autocontrainte.

Les analyses de N. Elias dans La civilisation des mours et La dynamique de l'Occident s'avèrent particulièrement utiles. N. Elias, tentant d'expliquer l'élévation du seuil de la sensibilité, montre comment, dans l'établissement de la société de cour, le raffinement des mœurs devient un «code distinctif du comportement». Les changements dans les mœurs sont alors marqués par un déplacement du seuil de la sensibilité, par une intériorisation des émotions et des contraintes touchant aux fonctions corporelles. Si les revues féminines sont bien l'un des lieux où peut s'effectuer un travail de civilisation, par le travail de relais des normes en usage opéré par les magazines, les

19. Les tickets de rationnement ont perduré jusqu'en 1949 pour certains produits. 
évolutions qu'on y observe illustrent bien cette intériorisation de la contrainte: vigoureusement exprimées par les revues, les différentes contraintes se font de plus en plus implicites.

Non que celles-ci, à la différence des attitudes en matière de sexualité qu'évoque Elias, soient marquées par une pudeur de plus en plus grande. Mais elles passent bien à l'arrière-plan, elles se situent dans le non-dit, ce qui semble suggérer leur intériorisation par les lectrices. Et si l'exigence se fait toujours plus forte en matière de minceur, il apparaît également que toutes les normes en matière de corpulence passent elles aussi de la contrainte sociale à l'autocontrainte: elles sont conçues comme allant de soi, dans un jeu de compétition sociale, ce qu'Elias décrivait dans sa théorie de la civilisation. Par son corps, il s'agit de se distinguer d'un autre moins bien situé socialement et plus gros que soi (Régnier et Masullo, 2009). Ce passage de la contrainte à l'autocontrainte repose tout d'abord sur la disparition du discours de la nécessité.

\section{a) La disparition du discours de la nécessité}

Dans les deux revues, les discours montrent une diminution des termes liés à la nécessité. Ainsi, dans Good Housekeeping, bien des termes de ce registre régressent au fil du temps. Il s'agit tout d'abord du vocabulaire injonctif: les verbes d'injonction «must» et «should», dont on a noté l'importance dans ce corpus, sont en forte diminution. Peu à peu, le ton du discours change: l'injonction venue de la revue est en diminution. Pour autant les règles ne disparaissent pas — en témoigne l'ampleur des textes recueillis dans les dernières décennies, qui s'intéressent justement à tous les conseils et règles en matière d'alimentation et de corpulence. Mais ces conseils et règles se présentent désormais comme allant de soi: ils sont intériorisés.

Ensuite, les termes indiquant la nécessité d'une adéquation aux règles nutritionnelles sont également en régression: tels «necessary», "essential(s)», "quantity», «requirement», "sufficient», "amount», «deficiency». Ces termes, qui énoncent une norme nutritionnelle (par exemple adopter un régime alimentaire qui procure une quantité suffisante de calcium et phosphore), sont de moins en moins employés. Il en va de même, enfin, pour les termes indiquant la «bonne alimentation » et l'adéquation entre les besoins du corps et ses dépenses énergétiques.

L'optique selon laquelle il s'agit d'assurer la quantité nécessaire des différents aliments ou nutriments, qui caractérise les premières années du corpus, s'efface devant un souci de qualité nutritionnelle. Au départ, il s'agit de couvrir des «besoin(s)», propres aux années 1930, aux États-Unis (3,3 pour «need»; 7,4 pour «needs») et aux années 1950 en France (2,5 pour «besoin»; 2,9 pour «besoins»). À ces «besoins» du corps étaient liées les notions de ration, de nutriment, de quantité nécessaire, en régression dans le corpus: désormais, ces besoins de base sont couverts.

Dans Modes et Travaux, le discours évolue également dans le sens d'une euphémisation. On passe en effet d'un discours injonctif, dont témoigne la fréquence des verbes «devoir» et «falloir», à un discours beaucoup plus modéré. Au fil du temps, le discours se nuance: il ne s'agit plus de donner des «règles» (propres aux années 1970, 3,4 pour 
le pluriel et 2,7 pour le singulier), mais des «conseils» (propres aux années 1990, 3,7). Les injonctions («il faut», «nous devons», «doit») finissent par disparaître après octobre 1982. On préférera alors le conditionnel «devrait», laissant — en apparence peut-être - plus de liberté à la lectrice: «Une alimentation idéale devrait donc comporter plusieurs prises alimentaires régulièrement réparties dans la journée » $(\mathrm{MT}$, 04/2003, p. 58).

Le discours semble se faire plus souple, en témoignent ces extraits liés aux exigences corporelles pour les femmes en maillot de bain. En 1950, Modes et Travaux édicte des normes particulièrement sévères à la fois dans le ton et dans le niveau d'exigence: «(...) avant de s'exhiber en maillot une femme doit être certaine de pouvoir se montrer souple, mince, gracieuse, sans bourrelets de graisse ni maigreur excessive» (MT, 05/1950, p. 60). En 2004, le discours est en apparence beaucoup moins normatif, avec un objectif présenté comme très accessible, à condition de suivre les conseils donnés: «Entraînement, alimentation, soins... il suffit de s'y mettre pour changer d'allure en un temps record. Voici la panoplie complète pour être superbe en maillot, corsaire ou minijupe» (MT, 06/2004, p.139). Mais sous une apparente liberté et facilité de moyens, le résultat - la perfection du corps - reste attendu et suggéré. Bien plus, dans les années 1950, les femmes — comme le suggère la citation — ont encore la possibilité, si elles ne sont pas conformes aux normes, de ne pas s'exhiber: à cette date, les Français découvrent tout juste les congés payés et entrent progressivement dans le temps des loisirs ${ }^{20}$. En 2004, il va de soi que la femme doit se montrer sur la plage. En cinquante ans, on est passé d'une vision esthétisante à une vision performative du corps.

De même, la position de la revue à l'égard de la lectrice évolue. Au début du corpus, il s'agit d'une position surplombante: la revue détient les savoirs en matière d'alimentation et détermine les règles à suivre, comme en témoigne la récurrence de l'expression à la deuxième personne du pluriel ( «vous devez», «vous allez»). Quand elle emploie la première personne du pluriel («nous devons»), la revue adopte une démarche pédagogique, et elle indique sa participation à une démarche vers une meilleure alimentation: "Une alimentation bien comprise est celle qui apporte aux différentes cellules du corps les éléments vitaux que chacune d'elles réclame. Nous devons apprendre à connaître la valeur et les propriétés de chaque substance alimentaire» (MT, 10/1952, p. 80)

Au fil du temps, la revue abandonne cette position en surplomb, se mettant au même niveau que la lectrice. La hiérarchie s'effacerait entre journaliste et lectrice, à qui la parole semble donnée.

20. La première loi sur les congés payés remonte à juin 1936, complétée par celle de 1956 qui institue les trois semaines de congés payés. 


\section{b) De la mère qui nourrit autrui à la femme centrée sur elle-même}

L'évolution du statut de la lectrice telle qu'elle est mise en scène dans les revues illustre en effet cette intériorisation de la contrainte. Dans les premières décennies du corpus, jusqu'aux années 1960, les revues s'adressent toutes deux à la lectrice mère de famille, celle qui a la responsabilité de nourrir autrui. Modes et Travaux évoque ainsi la «ménagère» et ses responsabilités de mère de famille en matière d'alimentation: "Notre santé, notre caractère, notre apparence, notre manière de penser, même, dépendent de la façon dont nous nous alimentons. De cette science, si importante, de la nutrition, la ménagère doit connaître les grandes lignes; elle tient entre ses mains le courage et la bonne humeur de toute la famille. Avec l'aide précieuse des services de l'Institut scientifique d'hygiène alimentaire, nous vous aiderons chaque mois à remplir la plus importante de vos missions» (MT, 10/52, p. 80).

Par l'emploi de la première personne du pluriel «nous», la revue indique maintenant qu'elle est partie prenante des efforts faits (supposés faits) par la lectrice, et elle marque une solidarité avec elle.

Mais ces termes de «ménagère», «Madame» «maman» sont en régression dans le corpus au fil du temps, alors que le pronom personnel «je» est de plus en plus utilisé. Celui-ci est ainsi propres aux années $2000(12,4)$, et, sur les 180 occurrences du «je», 124 apparaissent dans les années 2000. La lectrice passe du statut de ménagère et de mère, à celui de femme individualisée, qui parle désormais en son nom propre. Cette apparente subjectivité matérialise, dans le discours, l'intériorisation de la contrainte et l'individualisation des propos: «Je retrouve la ligne avec le régime wok!» (MT, 02/2005, couverture), «Nutrition. Je mange tout cru» (MT, 07/2007, p. 70).

Depuis quelques années, on assiste ainsi à une personnification de la lectrice: la lectrice est mise en scène et apparaît dans sa singularité et son individualisme, à travers un double procédé. D'une part, la revue donne la parole à la lectrice et la met en scène; d'autre part, elle présente des figures de femmes ayant réussi avec succès à perdre du poids, constituant ainsi des guides d'opinion et des figures d'identification positive. L'irruption de discours à la première personne du singulier accentue cette impression d'intériorisation forte de la contrainte: le «je» institue l'adhésion au modèle. Pour autant, la prise de parole, certes fictive, est particulièrement ambiguë. Qui parle en effet? La lectrice ou la journaliste qui se met à la place de la lectrice pour lui indiquer ce qu'il convient de faire?

Dans Good Housekeeping, le mouvement est exprimé de manière différente. Dans les premières années, la revue s'adresse, comme dans Modes et Travaux, à la mère de famille, à celle dont la responsabilité est de bien nourrir les membres du ménage ${ }^{21}$ : les enfants et l'époux. La revue écrit ainsi: "Avoir une alimentation adaptée pour les hommes de la famille est devenu de plus en plus important» $(02 / 69$, p. 183). Ce rôle — ou du moins le rappel de ce rôle - diminue au fil du temps: les «enfants», le mari

21. Sur le travail invisible que représentent les tâches alimentaires — planification, approvisionnement, préparation des repas —, voir DeVault, 1991. 
et les pronoms qui le désignent («him» «his») sont des termes en régression dans le corpus. De même, la figure de la mère s'efface, comme en France, au profit de la femme: «mother» est un terme en régression au fil du temps.

Mais contrairement à ce que l'on observait dans le corpus français, ce n'est pas le «je» qui prend la parole, c'est «you» et «yourself» qui marquent une progression. Ils indiquent un centrage de la revue sur la lectrice et ses préoccupations. Le «je», sans être spécifique ni dominant, n'est cependant pas absent. Quand il apparaît, il n’indique pas un discours prêté à la lectrice: il renvoie à la prise de parole de femmes (réelles ou fictives, mais qui se présentent comme réelles) qui racontent leur conquête victorieuse de la minceur.

\section{c) Le plaisir : une affirmation récente... mais jamais sans contrainte}

Dès lors, la place semble libre pour l'affirmation du plaisir. Alors que s'efface l'énonciation d'un déplaisir créé par la soumission à la nécessité, et parallèlement à cette intériorisation de la contrainte, le plaisir prend une place de plus en plus importante au fil du temps. Peut-on y voir l'apogée du mouvement vers l'individualisme et l'hédonisme qui s'est amorcé après les années 1960 (Mendras, 1988)?

Dans Modes et Travaux, le "plaisir» est un marqueur des années 2000 (5,6). Parallèlement, les verbes d'injonction diminuent en fréquence, de même que les occurrences de l'adjectif «pénible», qui, indiquant le déplaisir, disparaît à partir des années 1970. Quand il était évoqué, ce déplaisir faisait référence essentiellement à la pénibilité d'un régime. Évoquant par exemple un régime sans sel destiné à «celles qui gonflent » la revue précise: «Évidemment il est pénible et demande une certaine volonté» (10/49, p. 50). Au fil du temps, la revue au contraire s'attache à souligner que les régimes qu'elle propose ne sont précisément pas pénibles: on est passé d'une vision du régime restrictif et déplaisant, supposant de la volonté et de la contrainte, à une vision où le contrôle de l'alimentation est désormais lié au plaisir. Il ne s'agit donc pas d'un relâchement des contraintes: le souci de conformité à la minceur et les pratiques d'abstinence qui l'accompagnent font désormais partie du paysage. Dès lors, ils constituent une source de plaisir.

Aux États-Unis, où le plaisir est une notion moins centrale qu'en France, on ne constate pas la place prise par cet affect. L'association plaisir / contrainte d'un régime amaigrissant y est très précoce: «Maigrir avec plaisir» est le titre d'une brochure publiée par la revue en 1934. Cependant, il faut souligner dans le corpus américain l'expansion remarquable du terme «to love» : c'est celui qui connaît la plus forte progression au fil du temps dans tout le corpus américain, ce qui indique l'importance prise par une forme d'expression du plaisir. Elle désigne également l'ampleur prise par un discours d'emphase qui prend de plus en plus de place dans le corpus. En témoigne la disparition de certains termes qui indiquaient le plaisir gustatif, remplacés par des termes plus laudatifs, voire hyperboliques ${ }^{22}$ : «appétissant» disparaît après les années

22. Si l'on peut traduire ainsi une tendance américaine contemporaine, caractérisée par un overstatement du discours. 
1970, alors que les discours qui suivent évoquent des plats «préférés» ou «délicieux» (termes en progression), qui soulignent un degré croissant du plaisir.

Pour autant, dans Modes et Travaux comme dans Good Housekeeping, ce plaisir en augmentation ne se situe pas à l'opposé de la nécessité ou de la contrainte. Tout au contraire, il lui est indissolublement lié, et de plus en plus explicitement à mesure qu'il occupe plus de place. L'analyse des domaines d'application du plaisir montre le passage d'un plaisir opposé à la contrainte à un plaisir qui se situe désormais dans le respect de la contrainte. Faire une place au plaisir suppose-t-il de rappeler la contrainte, en soulignant les liens qui les attachent?

Dans le corpus français, dans les années 1930 et 1940, le plaisir est une sensation dont il faut se méfier, ou bien il est associé aux enfants — on évoque «quelques bonbons qui leur font tant plaisir» (MT, 02/1937). Le plaisir renvoie ici à une dimension régressive de l'alimentation, celle du sucrée.

Par la suite, le plaisir vient également caractériser des aliments ou types d'aliments dont la consommation fait plaisir mais qu'il faut limiter, par exemple le «fromage» ou les «corps gras». Le plaisir vient aussi caractériser l'excès, et dès lors il est condamné: «Manger salé, sucré, gras, ne pas faire d'effort, se faire plaisir représentent à plus ou moins long terme un danger pour la santé» (MT, 11/1979, p. 208). Du plaisir certes, mais point trop n'en faut. De même, dans Good Housekeeping, «love» est employé au départ pour signaler la restriction, voire la nécessaire répression des appétences: «Refusez ou consommez avec modération les aliments avec lesquels les enfants dodus aiment se remplir» conseille la revue à la jeune fille qui cherche à surveiller son poids (GH, 10/1945, p. 62).

L'évolution illustre un changement radical de perspective. Au moment où le plaisir prend sa place dans les discours, il change de registre: il est désormais associé à la perte de poids. Maigrir fait plaisir: c'est le «plaisir de s'affiner puis de rester mince [qui] prime sur la «bonne bouffe»» (MT, 05/1988, p. 56). Le plaisir réside dans la perte de poids qui vient compenser la perte des joies de la table. Et si, dans les années 1950, le régime alimentaire ou la perte de poids sont liés aux privations de la période de guerre, dans une société d'abondance, la restriction est différemment connotée car elle est choisie et non subie. Bien évidemment, les revues le soulignent, il s'agit bien de «maigrir sans se priver» mais la négation comporte une part d'affirmation: par définition, maigrir, c'est se priver de certains aliments ou plats, ceux en particulier qui font le plus plaisir.

Bien plus, c'est désormais dans la restriction et dans la conformité aux règles nutritionnelles qui permettent de maigrir que se trouve la source du plaisir : le régime en soi est un plaisir. Et le plaisir est maintenant intimement lié à la contrainte. S'il l'a toujours été parce qu'il le serait intrinsèquement, c'est en tout cas à ce moment-là que les discours les associent. S'imposer un régime était source de déplaisir et de frustration. Désormais, le régime — la contrainte — devient la source même du plaisir — ou du moins est présenté comme tel — suivant un mouvement plus général de place prise 
par les contraintes diététiques qui sont, aujourd'hui, devenues une vraie morale (Régnier et Masullo, 2009).

Ainsi, sur les 69 occurrences du "plaisir» dans les discours de Modes et Travaux depuis les années 1980, 38 désignent le régime ${ }^{23}$. Lié à la restriction et à la nécessité, il est désormais plus facilement autorisé: «maigrir plaisir» «privilégier le plaisir» «ce nouveau régime plaisir», «le régime $100 \%$ plaisir», tels sont quelques-uns des thèmes mis en avant par le magazine. De même, Good Housekeeping, à partir des années 1970, affirme le plaisir dans la restriction, voire dans l'inversion du déplaisir: «Apprenez à aimer les légumes que vous détestez» (07/2007, p. 158), enjoint la revue.

Les autres termes liés au plaisir dans le corpus américain le sont également à la restriction, comme l'adjectif «favorite» employé pour suggérer comment telle habitude alimentaire peut être pratiquée dans le cadre d'une diète particulière. À propos du snacking, « ( ...) beaucoup d'experts pensent que le snacking, l'habitude alimentaire préférée des Américains, peut vous aider à perdre du poids et à le perdre plus vite» (GH, 01/76, p. 158).

De la même façon, les termes «enjoy», qui indique le plaisir pris dans l'acte alimentaire, et «tasty», qui désigne un plaisir gustatif, montrent une évolution des associations. «Enjoy» est tout d'abord associé au plaisir pris à manger des repas équilibrés: «Notre santé bénéficiera de notre alimentation si nous savourons des aliments qui sont bons pour nous» (GH, 08/1941, p. 130). Au fil du temps, le terme est associé, de façon presque exclusive, au régime alimentaire, quel qu'il soit. La revue propose par exemple des «menus avec moins de calories que toute la famille aura plaisir à manger» $(\mathrm{GH}$, 04/1970, couverture).

Quant au terme «tasty», dans les années 1940, la revue l'associe souvent — certes pas exclusivement - à l'excès: «Voulez-vous vraiment une silhouette mince, svelte? Ou bien accordez-vous plus d'importance au plaisir de vous remplir de desserts fourrés à la crème ou d'appétissants petits pains et d'aliments savoureux de toute sorte?» (GH, 08/1946, p. 123). À partir des années 1960, le terme devient presque exclusivement associé à la contrainte: celle de tous les régimes que propose la revue: régime sans sel, régime contre la goutte, régime basses calories, régime contre le cholestérol ou régime sans viande. "Enjoy» enfin est presque exclusivement associé au régime alimentaire, quel qu'il soit.

Le plaisir devient alors une injonction et une norme: "Manger doit rester un plaisir» affirme Modes et Travaux (MT, 05/1989, p. 102). Cependant, les discours sur le plaisir ne sont pas exempts d'un rappel de la norme, tout au contraire. En témoigne l'usage du verbe «falloir» dans le corpus français dans les années 2000, associé au plaisir. Évoquant la fin des «régimes frustration», la revue française énonce: «Il faut retrouver le plaisir de manger quand on a faim» (MT, 02/2003, p. 28), «il faut se faire plaisir tout en mangeant équilibré» (MT, 04/2009, p. 60) dit Modes et Travaux. La

23. Les autres occurrences évoquent soit le "petit plaisir» transgressif d'un dessert, d'un carré de chocolat ou d'un plat allégé autorisés dans le cadre d'un régime; soit l'importance du plaisir dans une optique de nutrition générale ou de santé plus générale. 
satisfaction de la faim, la recherche de l'équilibre alimentaire sont supposées acquises: la priorité est désormais — «il faut», supposant que la lectrice en a le pouvoir — le plaisir. Good Housekeeping estime bon de rappeler: "Mais la nourriture, ce n'est pas seulement du carburant — ce devrait être aussi un plaisir» (GH, 03/2002, p. 139). L'ordre est inversé: le plaisir vient en second lieu, c'est un plus.

De même, l'usage de l'infinitif dans Modes et Travaux — «savoir marier santé et plaisir», «mincir en se faisant plaisir», «allier régime et plaisir»- sous-entend une injonction ( «il faut») implicite, mais qui se trouve bien présente dans ces appels au plaisir. Enfin, le plaisir peut être justement ce qui permet d'embrasser la nécessité, comme l'indique dans Good Housekeeping l'usage du verbe «to please»: plaire, c'est faire plaisir à autrui.

\section{CONCLUSION}

En analysant deux revues de la presse féminine française et américaine, il apparaît que l'expression du plaisir et de la nécessité y tient une place différente et variable au fil du temps. Le plaisir est une notion typiquement française, alors que les États-Unis se caractérisent par l'ampleur des discours sur la nécessité.

Dans les deux pays néanmoins, le plaisir est moins présent que la nécessité, quand il s'agit de conseils d'économie domestique et de la définition de la bonne alimentation qui les accompagne. La nécessité concerne des domaines divers selon les époques. Dans les premières années du corpus, cette nécessité est caractéristique de la période de guerre et d'après-guerre, marquée par des contraintes budgétaires fortes et la satisfaction de besoins alimentaires de base. Dans un contexte de société d'abondance et de consommation de masse, elle est remplacée par une nécessité plus neuve: injonctions diététiques et nutritionnelles, exigence de santé et de minceur.

Cette nécessité, explicitement formulée jusqu'aux années 1960, se transforme sur la période peu à peu en autocontrainte, et le ton des revues se fait beaucoup moins injonctif. On aurait là le reflet du processus de civilisation décrit par N. Elias: l'expression moindre des contraintes n'indique pas leur affaiblissement, mais tout au contraire leur intériorisation. Au risque, peut-être, que ceux qui ne les auraient pas intériorisées soient désormais exclus du jeu.

Le plaisir, moins américain que français, se formule moins aisément: on s'en méfie longtemps, il faut le canaliser. Cette réserve peut correspondre au refoulement d'un affect, dans le cadre du processus de civilisation décrit par N. Elias. Comment alors interpréter son irruption dans les années contemporaines en France, et d'une manière plus indirecte, aux États-Unis? Peut-on y lire un relâchement des contraintes? Une inversion dans le processus de civilisation? Il semble plutôt que le plaisir soit devenu une nouvelle norme. «Faites-vous plaisir!» est la nouvelle injonction, contradictoire en elle-même. C'est au moment où la contrainte se fait la plus forte, où l'on fournit des normes encore plus rigoureuses en matière de corpulence, que le plaisir est privilégié. Ce qui est moins paradoxal qu'il n'y paraît: l'affirmation du plaisir vient contrebalancer la rigidité et la force des normes édictées. Fausse liberté et hédonisme obligé 
peut-être, puisque la recherche du plaisir se transforme en axiome: des plaisirs non nécessaires, relevant en particulier de l'esthétique corporelle, ont au fil du temps acquis le statut de plaisirs nécessaires dans la distinction opérée par Épicure. Dès lors, le plaisir n'est jamais exprimé sans la nécessité qui l'accompagne, trouvant parfois sa source dans le respect de cette dernière, et témoignant des liens indissolubles du plaisir et de la nécessité.

\title{
RÉSUMÉ
}

L'article examine la place respective tenue par le plaisir et la nécessité, et leur articulation au fil du temps, à partir d'un corpus de conseils d'économie domestique de 1200 pages, issu de la presse féminine française et américaine (Modes et Travaux et Good Housekeeping) entre 1934 et 2010. Partant d'un examen des termes employés pour indiquer d'une part le plaisir, d'autre part la nécessité, il montre les évolutions, à la fois dans la place tenue par le plaisir et par la nécessité, et dans les domaines où l'un et l'autre s'expriment (passage des contraintes liées au budget et à la satisfaction de besoins alimentaires de base à des injonctions diététiques et individualistes ensuite). S'inscrivant dans les analyses de N. Elias dans La civilisation des mœurs et La dynamique de l'Occident, l'article met en évidence que l'expression de la nécessité et la place laissée au plaisir témoignent d'un travail de civilisation, dans lequel la contrainte implicite devient autocontrainte. L'article montre que le plaisir, plus récemment affirmé et mis en valeur, ne s'effectue jamais sans son articulation à la nécessité: un hédonisme, sans doute, mais contraint.

Mots clés: économie domestique, presse féminine, alimentation, corps, autocontrainte, hédonisme

\begin{abstract}
Based on a corpus of 1200 pages of home economics advice columns from American and French women's magazines (Modes et Travaux and Good Housekeeping) between 1934 and 2010, this paper analyzes the respective positions of pleasure and necessity, and their links over time. The paper begins with a lexicometric analysis of words relevant for two dimensions: on the one hand the idea of pleasure, on the other hand that of necessity. The paper highlights the evolutions regarding the relative positions of both ideas, and the domains in which they are expressed (budget constraints and basic needs followed by dietetic and individualistic constraints). In reference to Norbert Elias, the way to express the tension between necessity and pleasure shows a civilizing process, in which explicit constraint is transformed into internalized self-restraint. Pleasure, a recent value, is never expressed without its links with necessity-: hedonism, no doubt, but always under constraint.
\end{abstract}

Key words: home economics, women's magazines, food, body, self-restraint, hedonism

\section{RESUMEN}

Este artículo examina el respectivo lugar que ocupan el placer y la necesidad, y su articulación con el paso del tiempo, a partir de un corpus de consejos de economía doméstica de 1200 páginas, tomado de la prensa feminista francesa y estadounidense (Modes et Travaux y 
Good Housekeeping), entre 1934 y 2010. A partir de un análisis de los términos empleados para indicar, por una parte, el placer, y por otra, la necesidad, se muestran las transformaciones tanto del lugar que ocupan el placer y la necesidad, y las áreas donde se expresan el uno y la otra (las limitantes asociadas al presupuesto y a la satisfacción de las necesidades alimentarias de base con conminaciones dietéticas e individualistas). Inscrito en los análisis de N. Elias en La Civilización de las Costumbres y La Dinámica de Occidente, este artículo pone en evidencia que la expresión de la necesidad y el lugar dado al placer son testimonios de un trabajo de civilización, en donde la limitante implícita llega a ser autolimitante. El artículo muestra que el placer, más recientemente afirmado y valorado, no se realiza jamás sin su articulación a la necesidad: una expresión de hedonismo, sin duda, pero limitada.

Palabras claves: economía doméstica, prensa femenina, alimentación, cuerpo, autocoacción, hedonismo

\section{BIBLIOGRAPHIE}

Bentley, A. (2002), «Islands of Serenity: Gender, Race, and Ordered Meals during World War II», in C. Counihan (dir.), Food in the USA, Routledge, New York, p. 171-192.

Besnard, P. et C. Grange (1993), «La fin de la diffusion verticale des goûts?», L'année sociologique, nº 43 , p. 269-294.

Bonvoisin, S. M. et M. Maignien (1986), La presse féminine, Paris, Presses universitaires de France.

Boutaud, J. -J. (dir.) (2005), Scènes gourmandes. Marché, cuisine, table, Paris, Jean-Paul Rocher.

Boutaud, J.-J. et V. Madelon (2010), «La médiatisation du culinaire», Communication et langages, $\mathrm{n}^{\circ} 164$, juin, p. 33-40.

BRUEGEL, M. (2013), «Un distant miroir. La campagne pour l'alimentation rationnelle et la fabrication du "consommateur» en France au tournant du xx ${ }^{e}$ siècle», Actes de la recherche en sciences sociales, ${ }^{\circ} 199$, p. 29-45.

Damian-Gaillard, B., C. Frisque et E. Saitta (2009), «Le journalisme au prisme du genre: une problématique féconde», Questions de communication, $\mathrm{n}^{\circ} 15, \mathrm{p} .175-201$.

DARD, O. et A. J. LÜsEbrink (dir.) (2008), Américanisation et anti-américanismes comparés, Lille, Presses universitaires du Septentrion.

DePeCKer, T. (2014), La loi des tables. Quantification du besoin alimentaire et réforme des conduites de vie, $X I X^{e}-\mathrm{XX} X^{e}$ siècles, thèse pour le Doctorat en sociologie, Paris, EHESS.

DeVault, M. (1991), Feeding the Family. The Social Organization of Caring as Gendered Work, The University of Chicago Press.

Dupuy, A. (2013), Plaisirs alimentaires. Socialisation des enfants et des adolescents, Presses universitaires de Rennes.

Elias, N. (1975 [1939b]), La dynamique de l'Occident, Paris, Calmann Lévy.

— 1973 [1939a]), La civilisation des mours, Paris, Calmann Lévy.

Ferguson, P. P. (2004), Accounting for Taste. The Triumph of French Cuisine, Chicago, University of Chicago Press.

Fischler, C. (1990), L'homnivore, Paris, Odile Jacob.

Fischler, C. et E. Masson (2008), Manger. Français, Européens et Américains face à l'alimentation, Paris, Odile Jacob.

FourastiÉ, J. (2011 [1979]), Les Trente Glorieuses ou la révolution invisible de 1946 à 1975, Paris, Pluriel.

Galbraith, J. K. (1999 [1958]), The Affluent Society, London, Penguin Books.

GIET, S. (2005), Soyez libres!, C'est un ordre — Le corps dans la presse féminine et masculine, Paris, Autrement.

Goldstein, C. (2012), Creating Consumers: Home Economists in the 20th Century America, University of North Carolina Press. 
Iulio, S. (de) (2011), “De la peur de manquer” aux dangers de l'obésité: les discours publicitaires et la construction sociale des risques alimentaires", Le Temps des Médias, 2, n 17, p. 143-163.

Levenstein, H. (1993), Paradox of Plenty. A social History of Eating in Modern America, Oxford, Oxford University Press.

- (1988), Revolution at the table. The transformation of the American Diet, NewYork, Oxford, Oxford University Press.

Mendras, H. (1988), La Seconde Révolution française, Paris, Gallimard.

Régnier, F. et A. Masullo (2009), «Obésité, goûts et consommation. Intégration des normes d'alimentation et appartenance sociale», Revue française de sociologie, 50-4, p. 747-773.

RéGnier, F. (2004), L'exotisme culinaire. Essai sur les saveurs de l'Autre, Paris, PUF.

Stearns, P. (1997), Fat History. Bodies and Beauty in the Modern West, New York University Press.

Warde, A. (1997), Consumption, Food and Taste, London, Sage.

Warde, A., S. L. Cheng, W. Olsen and D. Southerton (2007), "Changes in the Practice of Eating. A comparative Analysis of Time-Use», Acta Sociologica, décembre, vol. 50, n 4, p. 363-385.

\section{Annexe 1 - Expressions du plaisir et de la nécessité dans Modes et Travaux, par décennie}

\begin{tabular}{|l|l|l|l|l|l|l|l|l|l|}
\hline & 1930 & 1940 & 1950 & 1960 & 1970 & 1980 & 1990 & 2000 & Total \\
\hline Plaisir(s) & 4 & 2 & 3 & 3 & 2 & 11 & 16 & 51 & 92 \\
\hline Nécessité & 1 & & 5 & & 2 & 2 & 1 & & 11 \\
\hline Nécessaire(s) & 2 & 4 & 14 & 3 & 7 & 7 & 8 & 11 & 56 \\
\hline Plaire & 1 & & 2 & & 1 & 1 & 1 & 1 & 7 \\
\hline Plaisant & & & 1 & & 1 & & & & 2 \\
\hline Aimer & 3 & 1 & 2 & 4 & 2 & 7 & 2 & 21 & 42 \\
\hline Devoir (présent) & 16 & 7 & 49 & 29 & 53 & 33 & 28 & 23 & 238 \\
\hline Falloir & 26 & 8 & 27 & 27 & 15 & 39 & 37 & 55 & 234 \\
\hline Devoir (conditionnel) & & & 4 & 1 & 3 & 1 & 4 & 4 & 17 \\
\hline Règles & 1 & & 6 & 5 & 22 & 18 & 8 & 10 & 70 \\
\hline Principes & 4 & & 2 & 6 & 3 & 16 & 11 & 5 & 47 \\
\hline Essentiels & 1 & & 4 & 7 & 3 & 3 & 8 & 28 & 54 \\
\hline
\end{tabular}


Annexe 2 - Expressions du plaisir et de la nécessité dans Good Housekeeping, par décennie

\begin{tabular}{|c|c|c|c|c|c|c|c|c|c|}
\hline & 1930 & 1940 & 1950 & 1960 & 1970 & 1980 & 1990 & 2000 & Total \\
\hline Pleasure(s) & 5 & 3 & 8 & 2 & 1 & 1 & 5 & 2 & 27 \\
\hline Necessity & 2 & & 4 & & & & & 1 & 7 \\
\hline Necessary & 36 & 19 & 17 & 16 & 13 & 6 & 2 & 2 & 111 \\
\hline To please & 1 & 1 & 1 & 2 & 2 & 1 & 1 & 1 & 10 \\
\hline Pleasant & & 2 & 5 & 5 & & & & & 12 \\
\hline To love & & 3 & 7 & 10 & 14 & 18 & 21 & 32 & 105 \\
\hline Must & 46 & 53 & 53 & 28 & 16 & 11 & 5 & 13 & 225 \\
\hline $\begin{array}{l}\text { Have to, } \\
\text { has to }\end{array}$ & 7 & 20 & 11 & 6 & 10 & 16 & 21 & 32 & 123 \\
\hline Should & ו & 127 & 99 & 72 & 46 & 41 & 36 & 38 & 530 \\
\hline Rules & 14 & 6 & 3 & 8 & 10 & 8 & 13 & 15 & 77 \\
\hline Principles & 15 & 1 & 3 & & 4 & 1 & 1 & & 25 \\
\hline Essentials & 29 & 39 & 27 & 16 & 26 & 18 & 11 & 8 & 174 \\
\hline
\end{tabular}

